\title{
Antinuclear Antibodies Status and Related Risk Factors in Nonpregnant Adult Indian Women
}

\author{
Barun Kumar Chakrabarty ${ }^{1}$, Prosenjit Ganguli ${ }^{2}$, Sougat Ray ${ }^{3}$, Manu Chopra $^{4}$
}

\begin{abstract}
Introduction: Antinuclear antibodies (ANA) are the most commonly evaluated non specific autoantibodies in clinical practice. Previous studies showed ANAs are frequently detected in healthy individuals and expression of ANAs are more common in women and older age groups. To explain the reason of sex differences differential chromosomal, hormonal and reproductive factors are postulated, but till now the association is not clearly understood. There is paucity of Indian data of ANA status amongst women. In this study we examined the relationship of ANA status with socio demographic and reproductive factors in a representative sample of non pregnant adult Indian women.

Aims and Objectives:

- Estimation of ANA status prevalence in non pregnant Indian women by enzyme linked immunosorbent assay (ELISA) technique.

- Effects of socio demographic and reproductive factors with ANA prevalence.

Materials and methods: This study was conducted in the zonal level hospital. Non pregnant adult women who had ever menstruated and voluntarily completed the socio demographic and reproductive history questionnaire were included in the study. Serum ANA screening test were performed for the detection of IgG class antibodies to ANA in human serum by ELISA.

Results: We analyzed data on socio demographic and reproductive factors in relation to serum ANA status in adult non pregnant females using SPSS statistical software. The values thus obtained statistically similar with those obtained in developed countries.

Conclusion: These findings highlight the importance of considering sociodemographic and reproductive history in studies of autoimmunity in women in Indian set up.

Keywords: Antinuclear antibodies (ANA), ELISA, Nonpregnant adult women, Risk factors

Indian Journal of Medical Biochemistry (2019): 10.5005/jp-journals-10054-0097
\end{abstract}

\section{INTRODUCTION}

Incidence of autoimmune disorders like systemic lupus erythematosus (SLE), rheumatoid arthritis (RA), and primary biliary cirrhosis (PBC) has been increasing worldwide. ${ }^{1}$ Auto antibodies to cellular constituents are considered to be a hallmark of screening and diagnosis of autoimmune disorders (AIDs). These immune markers are of increasing interest in clinical research based on their potential as diagnostic, therapeutic and etiologic indicators across spectrum of diseases. ${ }^{2}$ Several researches described reactivity to antinuclear antigens even in healthy individuals, but the factors resulting autoimmunity are not well understood. ${ }^{3,4}$ Antinuclear antibodies (ANA) are the most commonly evaluated nonspecific autoantibodies in clinical practice. ANA positivity found to be appeared well before clinical presentation of autoimmune disorders and higher titer of ANA being well correlated as higher risk factor for of the onset of AIDs. ${ }^{5,6}$ But it is found to be positive in $>20 \%$ healthy individuals due to its association with several non-autoimmune conditions like cancers, infection, drugs and environmental factors. ANA also have a higher prevalence in females compared to males. To explain the reason of gender differences various chromosomal, hormonal and reproductive factors are postulated, but till now the association is not clearly understood. ${ }^{7}$ For ANA screening procedure in resource constraints countries most widely used method is ANA Enzyme linked Immunosorbant assay (ELISA). The epidemiologic characteristics of AID varied worldwide and there is paucity of Indian data of ANA status amongst women. ${ }^{8,9}$ In our study we examined associations of socio demographic and reproductive factors with ANA prevalence among adult female participants in Ranchi.
${ }^{1}$ Classified Specialist, ${ }^{2}$ Senior Advisor, ${ }^{3}$ Professor, ${ }^{4}$ Classified Specialist, Pulmonary Medicine

${ }^{1}$ Department of Pathology, Base Hospital, Guwahati, Assam, India

${ }^{2}$ Department of Pathology, Command Hospital (EC), Kolkata, West Bengal, India

${ }^{3}$ Department of Community Medicine, Institute of Naval Medicine, INHS Asvini, Mumbai, India

${ }^{4}$ Department of Medicine, AH (R \& R), Delhi, India

Corresponding Author: Barun Kumar Chakrabarty, Classified specialist, Department of Pathology, Base Hospital, Guwahati, Assam, India, Phone: 8092658543, e-mail: bkcdc@yahoo.co.in

How to cite this article: Chakrabarty BK, Ganguli P, Ray S, Chopra M. Antinuclear Antibodies Status and Related Risk Factors in Non-pregnant Adult Indian Women. Indian J Med Biochem 2019;23(2):250-253.

Source of support: Nil

Conflict of interest: None

\section{Aims ANd Obj ectives}

- Estimation of ANA prevalence in non-pregnant Indian women in Ranchi

- Associations of socio demographic and reproductive factors with ANA prevalence among adult female population.

\section{Materials and Methods}

\section{Study Population}

All non-pregnant adult women who attended the one day health camp organized by 492 bedded zonal level hospital form the

() The Author(s). 2019 Open Access This article is distributed under the terms of the Creative Commons Attribution 4.0 International License (https://creativecommons. org/licenses/by-nc/4.0/), which permits unrestricted use, distribution, and non-commercial reproduction in any medium, provided you give appropriate credit to the original author(s) and the source, provide a link to the Creative Commons license, and indicate if changes were made. The Creative Commons Public Domain Dedication waiver (http://creativecommons.org/publicdomain/zero/1.0/) applies to the data made available in this article, unless otherwise stated. 
study population. 71 non pregnant adult women who had ever menstruated and voluntarily completed the socio demographic and reproductive history questionnaire were included in the study. Written informed consent was obtained from all participants. Women who are presently carrying or presented with history of urine $\beta$-HCG test positivity were excluded from study.

\section{Clinical Details}

Body mass index was calculated for all the participants based on measured height and weight. Specified socio demographic and reproductive factors data were collected by competent physician by personal interview. Socio demographic data included age, marital status, age at first live birth, native Indian state, history of use of oral contraceptive pills and breast feeding practice. Menstrual histories included age at menarche, age at last period, history of having regular menstrual cycle or at least one cycle in the past 12 months, and the reasons for menstrual irregularities or not having even one period in the past 12 months (i.e., recent pregnancy and breast feeding, menopause and known medical conditions or managements). Reproductive history included total number of pregnancies, abortions and live births. Participants were enquired about known medical history of hypertension, thyroid disease, joint pain and rheumatoid arthritis. We defined parous women as those who had at least one pregnancy and nulliparous was defined only by reporting history of no pregnancy test positivity. Participants were asked about hysterectomy with or without ovaries removed and ages at time of the surgery. In our study we defined postmenopausal status as women of $\geq 45$ years who had history the persistent cessation of menstrual cycle for at least 12 months and which is not due to either recent pregnancy and breast feeding practices or known medical or surgical conditions. Women who had a surgical menopause were considered premenopausal if they were younger than age 45 . Post-menopausal women who were 45 years and older considered to have natural menopause. Menopause type could not be identified in some women due to absence of natural or known medical/surgical causes.

\section{Laboratory Data}

$05 \mathrm{ml}$ of random venous blood was collected from each participant. Collected blood serum was separated and processed within maximum three hours of collection. Serum ANA screening test were performed on read well TOUCH microplate Analyzer using commercially available Calbiotech Inc. (CBI) ANA kit for the detection of IgG class antibodies to ANA in human serum by enzyme-linked immunosorbent assay. After processing of the samples following kit manual Optical Density (O.D.) was measured at $450 \mathrm{~nm}$ using ELISA reader within $15 \mathrm{~min}$. $\mathrm{Ab}$ (Antibody) Index of each determination was calculated by dividing the O.D. value of each sample by cut-off value which was obtained based on kit Calibrator Factor (CF). For test interpretation kit manufacturer guidelines were followed. Antibody Index $<0.9$ interpreted as No detectable ANA IgG by ELISA, 0.9-1.1 Index value interpreted as Borderline positive and index value $>1.1$ was interpreted as Detectable ANA IgG by ELISA. The serum samples were also evaluated for Rheumatoid factors (RF) and C-reactive protein (CRP). RF was evaluated by RHELAX -RF latex agglutination slide test qualitative method for detection of rheumatoid factors of the IgM class in the serum sample. The reagent is standardized to detect $\geq 10 \mathrm{IU} / \mathrm{mL} R F$. CRP was evaluated by RHELAX-CRP latex agglutination slide test for the detection of $\mathrm{C}$-reactive protein in the serum sample. The reagent is standardized to detect CRP concentrations greater than $0.6 \mathrm{mg} / \mathrm{dL}$.

\section{Analysis}

We have used Statistical Package for the Social Sciences (SPSS) version 19 to analyze the clinical and laboratory data obtained from non pregnant female population in Ranchi area. Study descriptive parameters were analysed. Analysis of variance (ANOVA) was used to compare the groups. Pearson correlation analysis was done to find correlation with ANA positivity. $P$ value $<0.05$ was considered as statistically significant while analysing data.

\section{Results}

The ANA test was positive in 07 women out of 71 participants with prevalence of $9.9 \%$. In our study group most of the women are from 04 states Jharkhand (40.8\%), Bihar (18.3\%), Kerala (12.7) and West Bengal (11.3\%). Mean age group of the studied population was $41.3 \pm 12$ years with age range of 21-71 years. Average age of menarche was $13.9 \pm 1.4$ years and BMI was $23.6 \pm 3.6$. Table 1 shows the mean age, menarche and BMI of studied population. $54.9 \%$ females of our study population were premenopausal and $52.1 \%$ had regular menstrual cycle. $45.1 \%$ of our study population was belonging to post-menopausal age group. ANA positive cases were from Jharkhand (42.9\%), West Bengal (28.6) and Bihar (28.6\%). The ANA positive and negative groups are age matched and mean age group of the ANA positive cases was $45.4 \pm$ 8.3.The mean BMI between the two groups were statistically significant and average BMI of ANA positive cases was $26.6 \pm 4$.9. Average age of menarche was $14.2 \pm 1$ years and there is no statistically relevant differences obtained amongst the groups. $14.3 \%$ of the post-menopausal women were ANA positive where as in premenopausal group its $8.3 \%$. None of the nulliparous women were showing ANA positivity. Number of childbirth is statistically well correlated with ANA positivity $(p=0.02)$. ANA positivity were showing statistically

Table 1: Study descriptive

\begin{tabular}{llllllll}
\hline \multirow{3}{*}{ Age } & & $n$ & Mean & Std. deviation & Minimum & Maximum & Significance \\
& ANA negative & 64 & 40.8 & 12.7 & 21 & 71 & \\
\multirow{4}{*}{ Menarche } & ANA positive & 7 & 45.4 & 8.3 & 35 & 60 & 0.354 \\
& Total & 71 & 41.3 & 12.3 & 21 & 71 & \\
& ANA negative & 64 & 13.8 & 1.5 & 11 & 20 & \\
BMI & ANA positive & 7 & 14.4 & .98 & 13 & 16 & 0.277 \\
& Total & 71 & 13.9 & 1.4 & 11 & 20 & \\
& ANA negative & 64 & 23.3 & 3.4 & 14.52 & 34.85 & \\
& ANA positive & 7 & 26.6 & 4.9 & 21.56 & 36.63 & 0.022 \\
& Total & 71 & 23.6 & 3.6 & 14.52 & 36.63 & \\
\hline
\end{tabular}


Table 2: Results of RA factor, CRP and ANA tests

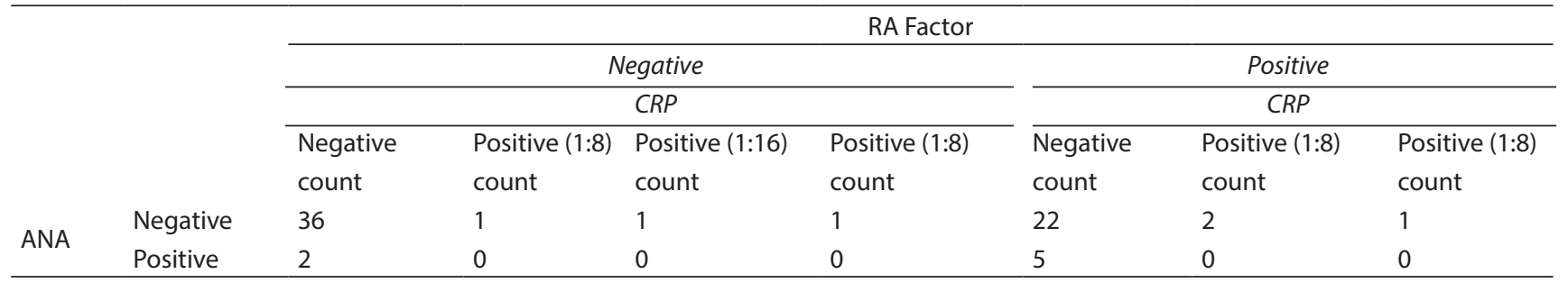

significant correlation with increasing body mass index $(p=0.02)$ and age of the first child birth of the participants $(p=0.01)$. None of the ANA positive cases had history of joint pain or thyroid disorders. Only one of them had history of hypertension. All the positive cases followed the breast feeding practices after child birth. None of them had history of oral contraceptive use. Out of 07 ANA positive cases 05 cases were exhibiting RF latex agglutination positivity and none of the cases were CRP positive. Table 2 shows summary of test results of RA factor, CRP and ANA tests amongst studied population.

\section{Discussion}

ANA are the most commonly used markers for autoimmunity and it is the easiest tool to be implemented as autoimmunity assessment at the population level. Antinuclear antibodies (ANA) are frequently detected in patients with systemic lupus erythematosus (SLE) and, less frequently, in other AIDs like Rheumatoid arthritis, Collagen vascular diseases, chronic liver diseases and systemic sclerosis (scleroderma). ANA bind to various nuclear antigens including DsDNA, SSDNA, RNP, Sm, SSA and SSB. ANA positivity frequency upsurges with age in apparently healthy people, especially in women older than age of 45 years. ANA ELISA is widely used as a preliminary screening tool for autoimmune diseases in developing countries like India. ${ }^{10}$

This is the first research report for detection of the prevalence of ANA in the non-pregnant female population in Ranchi area. ANA positivity prevalence in the US population aged 12 years and older is reported to be $13.8 \% .^{2}$ Study amongst Japanese revealed a prevalence rate of $9.5 \%$ at a cut of level of 1:100 dilutions, ${ }^{11}$ whereas for Indian population it is reported $12.3 \% .^{8}$ Data revealed from previous studies showed ANA positivity frequency in adults differs by geographic variation, sex and age factors. Our result showed the positivity rate amongst adult females is $9.9 \%$ and there is increased prevalence in eastern India than the southern Indian population. Some previous research studies suggest that aging is linked with autoimmunity and the prevalence of ANA is higher in the elderly population. ${ }^{12}$ Our study showed though the age factor was not significantly effects ANA positivity but With increased age and after the menopause the positivity rate increased amongst the studied population which indicates that female hormones like estrogen may perform an important factor in development of autoimmunity. ${ }^{13}$

The higher prevalence of ANA with increased BMI, in the present study may be explained by adipose tissue capability to produce pro-inflammatory cytokines ${ }^{14}$ and estrogen. ${ }^{15}$ However there are studies demonstrating inverse association of ANA frequency with obesity in western population. ${ }^{16}$ Additional research is needed to understand the cause of higher prevalence of autoantibodies amongst high BMI adult Indian females.

Our results suggest childbirth may be an important factor for the development of ANA in Indian women. There is an association of parity with ANA positivity demonstrated in this study in accordance to previous western population based studies. It is consistent with the concept that pregnancy increase the risk of autoimmunity. ${ }^{17,18}$ Pregnancy and childbirth make intense change in female hormonal level resulting in immune modulation and results in subsequent development of AIDs. ${ }^{19}$ Prolactin which is elevated during breast feeding also found to be associated with increased incidence of autoimmune diseases. ${ }^{20}$ But we observed no link of breast feeding with ANA positivity. Estrogen hormone receptors are expressed in most immune modulatory cells, but their ultimate effects is influenced by receptor type and may be a complex phenomenon. Therefore it is imperative that estrogen can influence antibody-mediated immunity. Our outcomes do not support the idea that exogenous hormone use increases the prevalence of ANA in women with a complex relation suggesting differences by menopause status.

Previous studies have concentrated pre-pregnancy autoimmunity effects on infertility and other adverse outcomes, ${ }^{21}$ our study demonstrated increasing age of the first child birth of the participants increase the possibilities of autoimmunity. Late age of menarche is associated with less lifetime menstrual cycles and lower oestrogen levels, ${ }^{22}$ so the perceived trend of nonsignificant association of late menarche age with ANA positivity was unexpected.

In our study none of the screened women who are positive for ANA were having symptoms of joint pain or thyroid disorders or pregnancy loss. They are also negative for inflammatory markers but 05 out them are positive for another autoimmunity marker (RA factor).Previous studies demonstrated patients who develop systemic autoimmune diseases, ANA can be detected years prior to clinical presentation, with predicting importance of the onset of clinical disease. ${ }^{23,24}$ But similar relationship not yet established between factors contributing to the progression from ANA-positivity to an autoimmune disease in healthy individual. Therefore the observation in the study required to be evaluated on larger cohorts. ${ }^{25}$

It is a small study on a small study population without comprehensive consideration of other factors such as occupation, genetic profile, and biochemical dynamics.The ideal gold standard of ANA assay done by immunofluorescence study on HEp-2 cells to detect IgG antibodies. ${ }^{26}$ Therefore our study is limited by shortfall of ELISA method and small of sample size.

\section{ConCLUSION}

Our study results highlight the need of large scale pan Indian studies to evaluate the role of sociodemographic and reproductive history in studies of development of autoimmunity in women in Indian set up. These aspects should be highlighted in mind by physicians while assessing ANA results and will serve as a useful baseline for future studies of autoimmunity. 


\section{References}

1. Feldman $\mathrm{CH}$, Hiraki LT,Liu J,et al. Epidemiology and socio demographics of systemic lupus erythematosus and lupus nephritis among US adults with Medic aid coverage, 2000-2004. Arthritis Rheum. 2013;65:753-763.

2. Satoh M, Chan EK, Ho LA, et al. Prevalence and sociodemographic correlates of antinuclear antibodies in the United States. Arthritis Rheum. 2012;64: 2319-2327.

3. Arbuckle MR, McClain MT, Rubertone MV, Scofield RH, Dennis GJ, James JA, et al. Development of autoantibodies before the clinical onset of systemic lupus erythematosus. N Engl J Med. 2003; 349:1526-1533.

4. Yadin O, Sarov B, Naggan L, Slor H, Shoenfeld Y. Natural autoantibodies in the serum of healthy women--a five-year follow-up. Clin Exp Immunol. 1989;75:402-406.

5. Wijeyesinghe U, Russell AS. Outcome of high titer antinuclear antibody positivity in individuals without connective tissue disease: a 10-year follow- up. Clin Rheumatol. 2008;27:1399-1402.

6. Eriksson $\mathrm{C}$, Kokkonen $\mathrm{H}$, Johansson $\mathrm{M}$, et al.Autoantibodies predate the onset of systemic lupus erythematosus in northern Sweden. Arthritis Res Ther. 2011;13:30-35.

7. Pennell LM, Galligan CL, Fish EN. Sex affects immunity. J Autoimmun. 2012;38:J282-J291.

8. Minz RW,Kumar Y,Anand S, et al.Antinuclear antibody positive autoimmune disorders in North India: anappraisal. Rheumatol Int. 2012;32:2883-2888.

9. Quintero OL, Amador-Patarroyo MJ, Montoya-Ortiz G, Rojas-Villarraga A, Anaya JM. Autoimmune disease and gender: plausible mechanisms for the female predominance of autoimmunity. J Autoimmun. 2012; 38:J109-J119.

10. Emlen W; O'Neill L Clinical significance of antinuclear antibodies: comparison of detection with immunofluorescence and enzymelinked immunosorbent assays. Arthritis Rheum 1997;40(9):1612-1618.

11. Hayashi N, Koshiba M, Nishimura K, et al. Prevalence of diseasespecific antinuclear antibodies in general population :estimates from annual physical examinations of resident so fasmalltownovera5yearperiod. Mod Rheumatol. 2008;18: 153-160.

12. Nilsson BO, Skogh T, Ernerudh J, Johansson B, Lofgren S, Wikby A, et al. Antinuclear antibodies in the oldest-old women and men. J Autoimmun. 2006;27:281-288.
13. Cacciapaglia F, Arcarese L,Rigon A, et al. Antinuclear antibodies prevalence in Filipinos migrated to Italy. Eur Rev Med Pharmacol Sci. 2008;12:267-270.

14. Ouchi N, Parker JL, Lugus JJ, Walsh K. Adipokines in inflammation and metabolic disease. Nat Rev Immunol. 2011;11:85-97.

15. Cleary MP, Grossmann ME. Mini review: Obesity and breast cancer: the estrogen connection. Endocrinology. 2009;150:2537-2542.

16. Gonzalez DA, De Leon AC, Rodriguez Perez MC, Coello SD, Gonzalez Hernandez A, Fuentes RC, et al. Inverse association between obesity and antinuclear antibodies in women. J Rheumatol. 2008;35:2449-2451.

17. Khashan AS, Kenny LC, Laursen TM, Mahmood U, Mortensen PB, Henriksen TB, et al. Pregnancy and the risk of autoimmune disease. PLoS One. 2011; 6 .

18. Jorgensen KT, Pedersen BV, Nielsen NM, Jacobsen S, Frisch M. Childbirths and risk of female predominant and other autoimmune diseases in a population-based Danish cohort. J Autoimmun. 2012; 38:J8.

19. Hughes GC. Progesterone and autoimmune disease. Autoimmun Rev. 2012;11:A502-1.

20. Shelly $S, B o a z M$, Orbach H. Prolactin and autoimmunity. Autoimmun Rev. 2012;11:A465-A467.

21. Carp HJ, Selmi C, Shoenfeld Y. The autoimmune bases of infertility and pregnancy loss. J Autoimmun. 2012;38:J266-J267.

22. Clancy KB, Klein LD, Ziomkiewicz A, Nenko I, Jasienska G, Bribiescas RG. Relationships between biomarkers of inflammation, ovarian steroids, and age at menarche in a rural polish sample. Am J Hum Biol. 2013;25:389.

23. Eriksson C, Kokkonen H, Johansson M, Hallmans G, Wadell G, RantapaaDahlqvist S. Autoantibodies predate the onset of systemic lupus erythematosus in northern Sweden. Arthritis Res Ther. 2011; 13:R30.

24. Olsen NJ, Li QZ, Quan J, Wang L, Mutwally A, Karp DR. Autoantibody profiling to follow evolution of lupus syndromes. Arthritis Res Ther. 2012;14:R174.

25. Mariz HA, Sato El, Barbosa SH, Rodrigues SH, Dellavance A, Andrade LE. Pattern on the antinuclear antibody-HEp-2 test is a critical parameter for discriminating antinuclear antibody-positive healthy individuals and patients with autoimmune rheumatic diseases. Arthritis Rheum. 2011;63:191-200.

26. Gonz'alez C; Martin T; Arroyo T; Garc'ia-Isidoro M; Navajo JA; Gonz'alez-Buitrago JM. Comparison and variation of different methodologies for the detection of autoantibodies to nuclear antigens (ANA). J Clin Lab Anal 1997;11(6):388-392. 